Pacific Journal of Mathematics

THE RIEMANN MAPPING THEOREM FOR PLANAR NASH
RINGS 


\section{THE RIEMANN MAPPING THEOREM FOR PLANAR NASH RINGS}

\section{G. EFroymson}

Recall $f: D \rightarrow R^{2}$ is a Nash function if $f$ is analytic and if there exists a polynomial $p_{f}(x, y, z) \not \equiv 0$ with $p_{f}(x, y, f(x, y)=0$ for all $(x, y)$ in $D$. We wish to show Theorem 1: If $D$ is a semi-algebraic simply connected, open domain in $R^{2}$, then $D$ is Nash isomorphic to $R^{2}$. This means that there exists a map $(x, y) \rightarrow\left(f_{1}(x, y), f_{2}(x, y)\right)$ where $f_{1}$ and $f_{2}$ are Nash functions on $D$ and the map is a Nash isomorphism of $D$ with $R^{2}$.

As a corollary, we obviously get $N_{D} \cong N_{R^{2}}$ where $N_{D}=\{f$ : $D \rightarrow R \mid f$ is a Nash function on $D\}$. Moreover, if $D$ is any connected semi-algebraic domain in $R^{2}$, it follows that $D$ is Nash isomorphic to $R^{2}$ minus $n$ points where $n=$ the number of holes in $D$. Here a domain is always considered to be open. The problem of classification of nonopen regions even in the plane is much more complicated and not settled as far as I know.

The proof of Theorem 1 is fairly constructive. One map used is the real two variable map corresponding to the complex variable map $z \rightarrow \sqrt{z}$. Using mainly this map, we can get $\partial D$ to be a piecewise polynomial curve, and so each piece of $\partial D$ is part of an analytic curve which never enters $D$. Then a rational map will map $D$ to the interior of the unit circle so $\partial D$ approximates the boundary of the unit circle. Then one maps to the upper half plane and $\partial D$ will be the graph of a function, still piecewise analytic, etc. Finally, we can constract a Nash function which will enable us to map $D$ to the upper half plane which is clearly Nash isomorphic to $R^{2}$.

1. The mappings. We will need the following maps and their conjugates by linear transformations.

(1) $z \rightarrow \sqrt{z}$. This is the map $(x, y) \rightarrow\left( \pm\left(\left(x+\left(x^{2}+y^{2}\right)^{1 / 2}\right) / 2\right)^{1 / 2}\right)$, $\left.\pm\left(-x+\left(\left(x^{2}+y^{2}\right)^{1 / 2}\right) / 2\right)^{1 / 2}\right)$ where the signs are determined by designating a curve $C$ from $P=(0,0)$ to $\infty$ which lies outside of $D$. The function will be discontinuous all along the curve $C$ which is alright since $C$ is outside $D$.

(2) The mapping $(x, y) \rightarrow\left(x, y\left(x^{2}+y^{2}\right)^{m}\right)$ which was used in [4] to straighten out cusps and will be used for the same purpose here. This map is analytic except at $P=(0,0)$.

(3) The mapping $(x, y) \rightarrow((\lambda x-2 y) / \mu, y)$ where $\lambda, \mu>0$. This maps the region between $y=\lambda x$ and the $x$-axis to the region 
between $y+\mu x=0$ and the $x$-axis and so opens up angles.
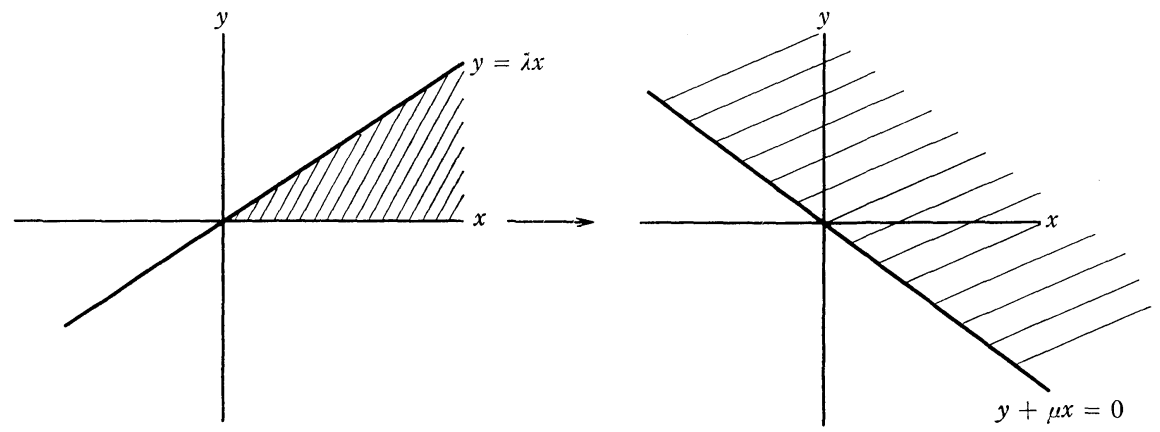

Note that the mapping $z \rightarrow \sqrt{z}$ can be used to "break" analytic ares as in [4]. Namely, if $y=0$ is to be broken at $(0,0)$ where we assume the negative $y$-axis is outside of $D$, consider the map $z \rightarrow \sqrt{z}$ as usual. Then the region $D$ will be mapped inside the right hand half plane and the image of the negative $x$-axis will be the positive $y$-axis while the positive $x$-axis is left fixed.

\section{Proof of Theorem 1 .}

TheOREM 1. Let $D$ be a semi-algebraic simply connected domain in $R^{2}$, then $D$ is Nash isomorphic to $R^{2}$.

Proof. Since $D$ is semi-algebraic, $\partial D$ is a union of polynomial arcs. So we first consider those ares which bound $D$ on both sides. Since $D$ is simply connected, any such arc is part of a curve $C$ running to $\infty$ outside of $D$. Now take $z \rightarrow \sqrt{z}$ where $P$ is the other end from $\infty$ of $C$. In the image, the rest of $\partial D$ will be no worse than before, but the double bounding arc will now be split into two isomorphic arcs each of which will bound $D$ only on one side. (See the diagram below.) Since there are only a finite number
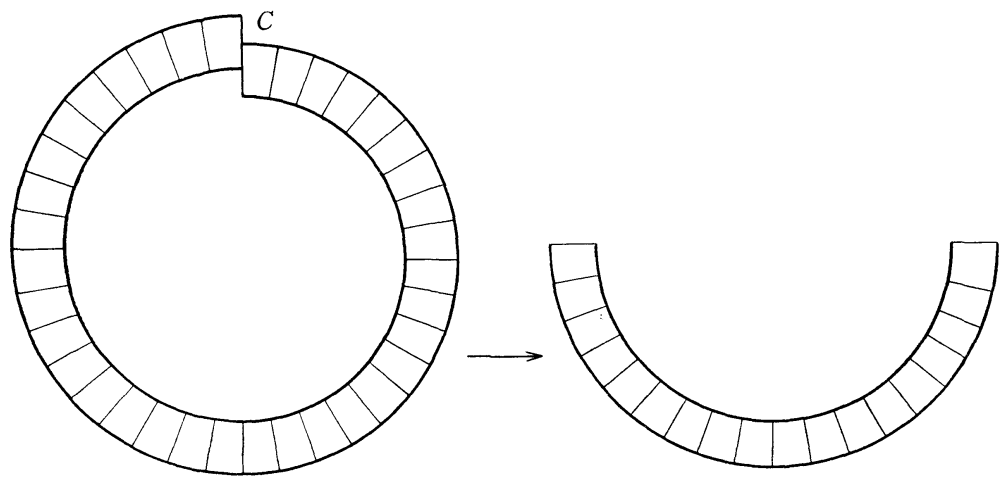
of double bounding arcs, in a finite number of steps we can remove them. Finally there may be some points where bounding ares intersect so the same type of behavior as above will occur only at one point. These points can be eliminated also as above.

Next, as in [4], we use map (2) to straighten out cusps. We briefly sketch the process. The object is to take a cusp like $y^{2}=x^{3}$ and change the angle from $0^{\circ}$ to $180^{\circ}$. So take the cusp with principal term $y^{m}-x^{n}$ where $m<n$. If $m$ is odd, the cusp will already have angle $180^{\circ}$. If $m$ and $n$ are both even, we get a factorization of $y^{m}-x^{n}$ so we can assume that $m$ is even and $n$ is odd. Consider the map: $(u, v) \stackrel{F}{\rightarrow}\left(f_{1}(u, v), f_{2}(u, v)\right)=\left(u, v\left(u^{2}+v^{2}\right)^{\alpha}\right)=$ $(x, y)$. I claim that this is a Nash isomorphism of $R^{2}-\{(0,0)\}$ to $R^{2}-\{(0,0)\}$. This is easily seen by considering $y=v\left(u^{2}+v^{2}\right)^{\alpha}$ as a function of $v$, and noting that $\partial y / \partial v=\left(u^{2}+v^{2}\right)^{\alpha}+2 \alpha v\left(u^{2}+v^{2}\right)^{\alpha-1}=$ $\left(u^{2}+v^{2}\right)^{\alpha-1}\left(u^{2}+v^{2}+2 \alpha v^{2}\right)>0$. Moreover, $v_{1}\left(u^{2}+v_{1}^{2}\right)=v_{2}\left(u^{2}+v_{2}^{2}\right)$ clearly implies that $v_{1}$ and $v_{2}$ have the same sign. So assume that $v_{i}>0$, and we see that $v_{1}=v_{2}$ and so the map is one-to-one. That the map is onto is clear.

Now suppose that we have $y^{m}-x^{n}$ as the dominant term in $p(x, y)$. Then $F^{*}(p(x, y))$ will have dominant terms $\left(v\left(u^{2}+v^{2}\right)^{\alpha}\right)^{m}-u^{n}$. To find the dominant term, we look at the Newton polygon and find that it is $v^{(2 \alpha+1) m}-u^{n}$. Now if, as above, $m$ is even and $n$ is odd, we find that for $\alpha$ large enough, this cusp will have angle $180^{\circ}$ (from the $u^{n}, n$ odd, term).

Next, we wish to "break" all the analytic arcs of $\partial D$ so they do not enter $D$. So let $C$ be an analytic arc of $\partial D$ and let $P$ be a boundary point of $C$ where it ceases to be on $\partial D$. That is, on one side of $P, C$ is part of $\partial D$ and on the other it is not. Now from $P$ we can run an arc $C^{\prime}$ to $\infty$ so $C^{\prime}$ lies outside $D$. Then, when we make the transformation $z \rightarrow \sqrt{z}$ centered at $P$, we break $C$ as we broke $y=0$ in the example. This works since the angle $C$ makes at $P$ will be $180^{\circ}$ even if $P$ is a cusp of $C$.

Now by mapping, if necessary, some point off $\bar{D}$ to $\infty$ we can assume that $\partial D$ is a closed curve. By the last procedure, all angles $>$ $180^{\circ}$ on $\partial D$ will have been eliminated. Now keeping in mind transformation (3), we see that we can map $D$ diffeomorphically to the inside of the unit circle so that all the corners of $\partial D$ lie on the unit circle. This map can be extended to a diffeomorphism of $R^{2}$ with itself so that the map is the identity at $\infty$ by [5], p. 180.

We next find a rational approximation to this map which takes $D$ to the inside of the unit circle with $\partial D$ having all its corners on the unit circle. We also want $\partial D$ to have smooth points at $\pm i$ and to approximate the unit circle closely enough so that the following 
circles cut $\partial D$ in only two points. These circles are obtained from the map $\varphi: R^{2} \rightarrow R^{2}$ which is given in complex coordinates by $z \rightarrow$ $(-i)(z-i) /(z+i)$, i.e., a Moebius transformation. The circles we want are the images of the circles $r=\left(x^{2}+y^{2}\right)^{1 / 2}=$ constant under the above map. So these circles are centered on the imaginary axis and always include in the interior $+i(r>1)$ or $-i(r<1)$; except for $r=1$, which has image the real axis. So approximating the unit circle closely by $\partial D$ will ensure that $\partial D$ cuts each of these circles in only two points.

Now, when we follow our rational map by the Moebius transformation $\varphi$ : we find:

(1) $D$ is contained in the upper half plane.

(2) $\partial D$ is a piecewise polynomial arc with two ends.

(3) Any analytic arc $C$ which contains a piece of $\partial D$ has $C \cap$ $D=\varnothing$.

(4) Any circle $x^{2}+y^{2}=r^{2}$ intersects $D$ in only two points, one on each side of the $y$-axis.

Of these properties (1) and (2) are clear, (3) follows from our construction.

Now for each analytic arc $C_{i}$ on $\partial D$, let $C_{i}^{\prime}$ be the analytic closure of $C_{i}$. Then since $C_{i}$ is contained in the zero set of a polynomial, we have by [4], Theorem 1, that there exists a Nash function $q_{i}(x, y)$ vanishing in $R^{2}$ only on $C_{i}^{\prime}$. By construction, $C_{i}^{\prime}$ does not intersect $D$, so $q_{i} \neq 0$ on $D$. So we can change sign if necessary to insure $q_{i}>0$ on $D$. Now set $q=\Pi q_{i}$. The Nash function $q$ is $>0$ on $D$ and vanishes $\partial D$.

Next let $h=q^{2} /\left(q^{2}+\varepsilon\right)=\left(1+\varepsilon q^{-2}\right)^{-1}$ with $\varepsilon(x, y)$ a function to be chosen. Then

$$
\begin{gathered}
\partial h / \partial y=-\left(1+\varepsilon q^{-2}\right)^{-2}\left((\partial \varepsilon / \partial y) q^{-2}=2 \varepsilon q^{-3}(\partial q / \partial y)\right) \\
(\partial h / \partial y) h=(2 \varepsilon(\partial q / \partial y) / q-\partial \varepsilon / \partial y) /\left(q^{2}+\varepsilon\right) .
\end{gathered}
$$

We wish to choose $\varepsilon$ so as to make $(\partial h / \partial y) / h>-2 / r>-2 / y$. To do this, we fix $r$ and consider the function $(\partial q / \partial y) / q^{3}$ on $x^{2}+y^{2}=r^{2}$, $(x, y)$ in $D$. This function will have a minimum since $q=0$ on $\partial D$, $q>0$ inside $D$, and by property (4) the circle $x^{2}+y^{2}=r^{2}$ will cut $\partial D$ in only two points, one on each side of $x=0$.

By the Tarski Seidenberg principle, we see that $\varphi(r)$ is piecewise algebraic so that there exists a constant $C>0$ and an integer $m$ so that $\varphi(r)>-C-r^{m}$.

Now let $\varepsilon=1 / C\left(2+r^{n}\right)$, where, for now, $n \geqq 2 m$. Then, noting that $\partial \varepsilon / \partial y<0$ for $y>0$, we see that

$$
\begin{gathered}
(\partial h / \partial y) / h>2 \varepsilon(\partial q / \partial y) / q^{3}>2 \varepsilon \varphi(y)>2 \varepsilon\left(-C-r^{m}\right) \\
=-2\left(C+r^{m}\right) / C\left(2+r^{n}\right)>-2 / r \geqq-2 / y
\end{gathered}
$$


since $\left(C+r^{m}\right) /\left(2+r^{n}\right) C<1 / r$ by the choice $n \geqq 2 m$.

We also need to choose $n$ large enough so that $h=q^{2} /\left(q^{2}+\varepsilon\right)$ is bounded from below as $y \rightarrow \infty$, i.e., $q^{2} /\left(q^{2}+\varepsilon\right)>1 / N$ for some large $N$ and all large enough $y$. But this is equivalent to the inequality $q^{2}>\varepsilon / N-1$. And by the Tarski-Seidenberg principle again, we have that $q^{2}>\lambda / r^{s}$ as $y \rightarrow \infty$ for some constant $\lambda>0$, and some integer $s>0$. So choose $n>s$ and we are okay.

Now let $g=y^{2} h$. Then $g$ vanishes on $\partial D$ and $g$ does not vanish inside $D$. Moreover $\left.\partial g / \partial y=2 y h=y^{2}(\partial h / \partial y)=y^{2} h((\partial h / \partial y) / h)+2 / y\right)>0$ on $D$. Now we map $(x, y) \stackrel{\psi}{\rightarrow}(x, g(x, y))$. Then $\psi$ maps $D$ into the upper half plane and we claim in fact $\psi$ maps $D$ isomorphically onto the upper half plane. For $\psi$ is one-to-one since each $g(x, y)$ is an increasing function of $y$ on $D$. Moreover, the inverse Nash map exists everywhere locally since its Jacobian is $\left|\begin{array}{cc}1 & 0 \\ \partial g / \partial x & \partial g / \partial y\end{array}\right| \neq 0$ anywhere on $D$. Moreover, the map is onto since, even through $q^{2} /\left(q^{2}+\varepsilon\right)=h$ is bounded, $g=y^{2} h$ won't be bounded as a function of $y$, ( $x$ fixed).

Finally, it is obvious that the upper half plane is isomorphic to $R^{2}$ since the map $(x, y) \rightarrow\left(x,\left(y^{2}-1\right) / y\right)$ will accomplish this isomorphism.

\section{Corollaries of Theorem 1 .}

THEOREM 2. Let $D$ be a semi-algebraic region (open still) with $n$ holes. Then $D$ is Nash isomorphic to $R^{2}$ minus $n$ points.

Proof. Let $D$ be a semi-algebraic connected domain in $R^{n}$. Let $R^{2} \cup\{\infty\}$ be the one point compactification of $R^{2}$. Then there are only a finite number of connected components of $R^{2} \cup\{\infty\}-D$. We define a hole in $D$ to be any of these components except the one containing $\{\infty\}$. We wish to map $D \stackrel{\cong}{\rightrightarrows} R^{2}-\{n$ points $\}$ where $n$ is the number of holes in $D$. First consider the component $S$ of $R^{2} \cup\{\infty\}-D$ containing $\{\infty\}$. Then, if $S=\{\infty\}$, we do nothing, and if not we $\operatorname{map} R^{2}-S \stackrel{\cong}{\rightrightarrows} R^{2}$ using Theorem 1 .

Now consider any hole $H$ of $D$ which is bigger than one point. Let $P$ be a point in $H$ and map $P=(a, b)$ to $\infty$ by $(x, y) \stackrel{\phi}{\rightarrow}$ $\left(x /(x-a)^{2}+(y-b)^{2}, y /(x-a)^{2}+(y-b)^{2}\right)$. Then map $R^{2}-\phi(H) \rightarrow R^{2}$ by a map \& using Theorem 1. Finally, follow this map by a map like $\phi$ which maps $\psi \circ \phi(\infty)$ to $\infty$ and we see that $H$ is mapped to a point hole in the image of $D$. By induction on the number of nonpoint holes, we complete the proof.

TheOREm 3. The nullstellensatz for $N_{D}$. Let $D$ be a planar 
connected semi-algebraic region (open). Let $\mathscr{P}$ be a prime ideal of $N_{D}$, the ring of Nash functions on $D$. Then $A_{D} / \mathscr{P}$ is orderable if and only if $I(V(P))=\mathscr{P}$. Here $V(\mathscr{P})=\{(a, b)$ in $D: f(a, b)=0$ for all $f$ in $\mathscr{P}\}$. And $I(V(\mathscr{P}))=\left\{g\right.$ in $N_{D}: g(a, b)=0$ for all $(a, b)$ in $V(\mathscr{P})\}$.

Proof. By Theorem 2, $D$ is Nash isomorphic to $R^{2}$ minus $n$ holes. So if the holes are the points $\left(a_{i}, b_{i}\right), i=1, \cdots, n$; then $D=$ $\left\{(x, y)\right.$ in $R^{2}:\left(x-a_{i}\right)^{2}+\left(y-b_{i}\right)^{2}>0$ for $\left.i=1, \cdots, n\right\}$. Now this is a region for which the nullstellensatz has been proved [2], [3]. (In [2] the nullstellensatz is proved only for planar regions of the restricted type and in [3] for higher dimensional regions of restricted type). The nullstellensatz is also known to be true for higher dimensional domains if one combines [3] with a proof (unpublished) by Shiota of Lemma 6 of Mostowski [6]. (This is explained in more detail in [1].)

\section{REFERENCES}

1. J. Bochnak and G. Efroymson, Real algebraic geometry and the Hilbert 17 th problem, Math. Ann., 251 (1980), 231-241,

2. G. Efroymson, A nullstellensatz for Nash rings, Pacific J. Math., 54 (1974), 101-112.

3. - Substitution in Nash functions, Pacific J. Math., 63 (1976), 137-145.

4. - Nash rings on planar domains, Trans. Amer. Math. Soc., 249 (1979), 435-445.

5. M. Hirsch, Differential Topology, Springer Verlag, New York, Berlin, Heidelberg, 1976.

6. T. Mostowski, Some properties of the ring of Nash functions, Annali della Scuola Normale Sup. di Pisa III, 2 (1976), 245-266.

Received June 26, 1978.

University of New Mexico

Albuquerque, NM 87131 


\section{PACIFIC JOURNAL OF MATHEMATICS}

\section{EDITORS}

DONALD BABBITT (Managing Editor)

University of Galifornia

Los Angeles, California 90024

Hugo RossI

University of Utah

Salt Lake City, UT 84112

C. C. MOORE AND ANDREW OGG

University of California

Berkeley, CA 94720
J. DUGUNDJI

Department of Mathematics University of Southern California Los Angeles, California 90007

R. Finn AND J. Milgram Stanford University Stanford, California 94305

\section{ASSOCIATE EDITORS}

R. ARENS

E. F. BECKENBACH

B. H. NEUManN

F. WOLF

K. YOSHIDA

\section{SUPPORTING INSTITUTIONS}

UNIVERSITY OF ARIZONA

UNIVERSITY OF BRITISH COLUMBIA CALIFORNIA INSTITUTE OF TECHNOLOGY

UNIVERSITY OF CALIFORNIA

MONTANA STATE UNIVERSITY

UNIVERSITY OF NEVADA, RENO

NEW MEXICO STATE UNIVERSITY

OREGON STATE UNIVERSITY
UNIVERSITY OF OREGON

UNIVERSITY OF SOUTHERN CALIFONIA

STANFORD UNIVERSITY

UNIVERSITY OF HAWAII

UNIVERSITY OF TOKYO

UNIVERSITY OF UTAH

WASHINGTON STATE UNIVERSITY

UNIVERSITY OF WASHINGTON 


\section{Pacific Journal of Mathematics}

\section{Vol. 92, No. $1 \quad$ January, 1981}

Michael E. Adams and J. Sichler, Lattices with unique complementation . ....1

Walter Allegretto, Positive solutions and spectral properties of second order

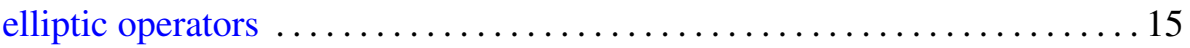

Philip J. Boland and Sean Dineen, Holomorphy on spaces of distribution . . 27

Duncan Alan Buell, Philip A. Leonard and Kenneth S. Williams, Note on

the quadratic character of a quadratic unit $\ldots \ldots \ldots \ldots \ldots \ldots \ldots \ldots \ldots$

Herbert Busemann and Bhalchandra B. Phadke, Two theorems on

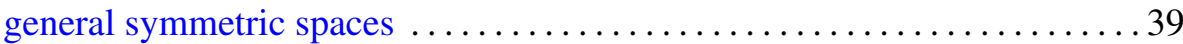

Emeric Deutsch, Bounds for the Perron root of a nonnegative irreducible

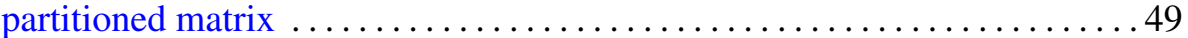

Charles F. Dunkl, A difference equation and Hahn polynomials in two

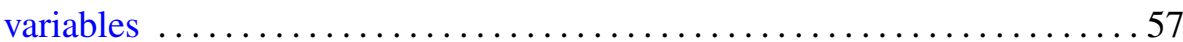

Gustave Adam Efroymson, The Riemann mapping theorem for planar Nash

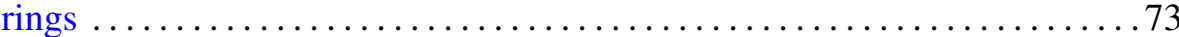

John Albert Fridy and Robert Ellis Powell, Tauberian theorems for matrices generated by analytic functions

Denton Elwood Hewgill, John Hamilton Reeder and Marvin Shinbrot,

Some exact solutions of the nonlinear problem of water waves .......887

Bessie Hershberger Kirkwood and Bernard Robert McDonald, The

symplectic group over a ring with one in its stable range

Esther Portnoy, Transitive groups of isometries on $H^{n}$

Jerry Ridenhour, On the sign of Green's functions for multipoint boundary

value problems

Nina M. Roy, An $M$-ideal characterization of $G$-spaces

Edward Barry Saff and Richard Steven Varga, On incomplete

polynomials. II

Takeyoshi Satō, The equations $\Delta u=P u(P \geqq 0)$ on Riemann surfaces and

isomorphisms between relative Hardy spaces

James Henry Schmerl, Correction to: "Peano models with many generic classes"

Charles Madison Stanton, On the closed ideals in $A(W)$

Viakalathur Shankar Sunder, Unitary equivalence to integral operators

Pavel G. Todorov, New explicit formulas for the $n$th derivative of composite functions

James Li-Ming Wang, Approximation by rational modules on boundary sets

Kenneth S. Williams, The class number of $Q(\sqrt{p})$ modulo 4 , for $p \equiv 5$

$(\bmod 8)$ a prime 